\title{
THE IMPACT OF LOCOREGIONAL TREATMENT ON SURVIVAL OF PATIENTS WITH PRIMARY METASTATIC BREAST CANCER
}

\author{
R. Liubota, *, V. Cheshuk ${ }^{1}$, R. Vereshchako ${ }^{1}$, O. Zotov ${ }^{1}$, V. Zaichuk ${ }^{1}$, N. Anikusko', I. Liubota ${ }^{2}$ \\ ${ }^{1}$ Department of Oncology, O.O. Bogomolets National Medical University, Kyiv 03115, Ukraine \\ ${ }^{2}$ Municipal City Clinical Oncological Centre, Kyiv 03115, Ukraine
}

\begin{abstract}
The aim of the study was to investigate the impact of primary tumor locoregional treatment (surgery or/and radiotherapy) on overall survival in patients with primary metastatic breast cancer (PMBC). Materials and Methods: This retrospective study included 295 women aged from 23 to 76 years with PMBC. Among the 295 patients, the effect of locoregional treatment of primary tumor on survival outcomes was evaluated in 177 women with distant metastases at diagnosis of breast cancer. 35 patient received breast surgery (group 1), 95 patients with PMBC - radiotherapy (group 2) and 47 patients - combination of breast surgery and radiation (group 3). The remaining 118 patients didn't receive surgery or/and radiotherapy (group 4). All patients received systemic cytotoxic chemotherapy. Results: The groups of patients with PMBC did not differ significantly by age, menstrual function, ER status, Her2 receptor status, site of metastasis and number of metastatic lesions. 2- and 5-year overall survival in patients of group 1 was 54 and 32\%, group $2-47$ and $8 \%$, group $3-$ 73 and $18 \%$, whereas in patients from group $4-26$ and $9 \%$, respectively. The median survival of patients who underwent surgery was 36 months, patients with PMBC who received radiotherapy - 24 months, patients who obtained combination of breast surgery and radiation - 30 months vs 18 months in patients who did not undergo primary tumor locoregional treatment. Conclusions: The results of this study showed a favourable effect of locoregional treatment in patients with PMBC.
\end{abstract}

Key Words: primary metastatic breast cancer, surgery, radiotherapy, survival.

Breast cancer $(B C)$ is one of the most common cancers among women worldwide. In 2012, BC incidence was 43.3 per 100,000 female population, $1,676,633$ new cases and 521,907 deaths from this disease were registered worldwide, which accounted for $25.2 \%$ of cases and $14.7 \%$ of deaths among all cancers in women [1]. In Europe and United States of America 5-10\% of patients of BC are diagnosed with primary metastatic disease and median survival ranges from 18 to 24 months [2]. According to the National Cancer Registry of Ukraine in 2014 7.0\% of patients were diagnosed with primary metastatic breast cancer (PMBC) [3]. Treatment of metastatic BC is palliative and symptomatic and must prolongate survival, control tumor burden, reduce cancer-related symptoms and improve quality of life. In recent years, the approach for the management of PMBC has been revised.

The value of the locoregional treatment (LRT) of the primary tumor and its impact on distant metastasis and survival is controversial. Currently, surgical treatment in this group of patients is used only to prevent and/ or treat local complications. However, results of many retrospective population-based trials demonstrated that an intensified multidisciplinary approach combining systemic therapies with surgery $[4,5]$, radiation, and regional chemotherapy may not only prevent local complications, but prolong survival of such patients similarly to the benefits observed in renal, colorectal, ovarian and gastric carcinoma [6-10]. The aim of this study was to investigate the impact of primary tumor

Submitted: November 14, 2016.

${ }^{*}$ Correspondence: E-mail: lyubota@ukr.net

Abbreviations used: $\mathrm{BC}$ - breast cancer; ER - estrogen receptor; LRT - locoregional treatment; OS - overall survival; PMBC - primary metastatic breast cancer; PR - progesterone receptor.
LRT (surgery or/and radiotherapy) on overall survival (OS) in patients with PMBC.

\section{MATERIALS AND METHODS}

The study included 295 women with PMBC aged from 23 to 76 years (median age $55 \pm 11$ years) who received treatment at the clinic of the Department of Oncology of the O.O. Bogomolets National Medical University based at the Kyiv Municipal Clinical Oncological Center from 2004 to 2006. All women with histologically proven $\mathrm{BC}$ and whose distant metastases are discovered at their first admittance were included in this study (in total, 295 patients). The diagnosis was defined according to the International TNM-classification ( $6^{\text {th }}$ edition 2002). Patients with isolated involvement of ipsilateral supraclavicular lymph nodes or contralateral lymph nodes metastases were excluded from this study.

Among the 295 patients with PMBC, the effect of LRT of primary tumor on survival outcomes was evaluated in 177 women with distant metastases at diagnosis of BC. 35 patient received breast surgery (group 1), 95 patients - radiotherapy (group 2), and 47 patients - combination of breast surgery and radiotherapy (group 3). The remaining 118 patients didn't receive surgery or/and radiotherapy (group 4). The study was approved by the Local Medical Ethics Committee. All patients gave their written informed consent before inclusion in this study. Patients from group 2 and 3 received radiation therapy delivered by a five-field technique. Field for conventional radiation treatment of the breast/chest wall: medial and lateral tangential fields of the whole breast are irradiated. Upper bound - lower edge of the clavicular head, i.e. lower edge of the first rib, lower bound $-1-2 \mathrm{~cm}$ inferior to the breast skin folds, medial margin — body midline 
and lateral margin - middle axillary line or posterior axillary line. The target dose was a median absorbed dose of 50 Gy in 25 fractions in 5 weeks. All patients received systemic cytotoxic chemotherapy.

Statistical analysis. Statistical significance of differences between the treatment group was evaluated by ANOVA and analysis of contingency tables $2 x K$ type. Differences were considered statistically significant at a significance level $(p)$ lower than 0.05 . Survival was analyzed using the Kaplan - Meier method comparing the elapsed time in months from diagnosis (start of the study), to death (critical event) due to BC progression, but not other causes. All statistical calculations were performed using Statistica 6.0 program.

\section{RESULTS}

The Table shows the clinical baseline characteristics of the patients. The study groups did not differ significantly by age, menstrual function, estrogen receptor (ER) status, Her2/neu receptor status, site of metastasis and number of metastatic lesions, indicating the equal distribution of patients in groups on the above criteria.

Table. Clinicopathological characteristics of BC patients

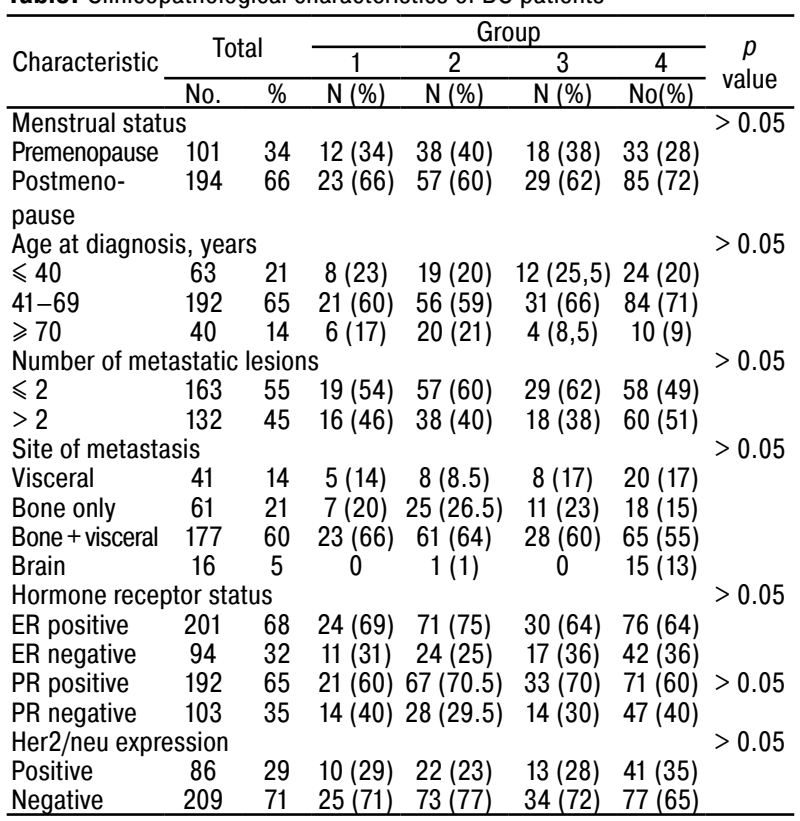

The median follow-up time of the whole population was 28 months (in a range 3-75 months). The Figure shows survival curves of PMBC patients from study groups according to treatment type.

2- and 5-year OS in patients of group 1 was 54 and $32 \%$, group $2-47$ and $8 \%$, group $3-73$ and $18 \%$, whereas those of patients of groups 4 were 26 and $9 \%$, respectively. The median survival for patients who underwent surgery was 36 months, patients who received radiotherapy -24 months, patients who were treated by combination of breast surgery and radiation was 30 months vs 18 months in patients who did not receive primary tumor LRT (surgery or/and radiotherapy). That shows the positive impact of LRT on the prognosis of patients with stage IV BC.

\section{DISCUSSION}

The PMBC should be considered as a terminal grade of chronic disease, and systemic cytotoxic therapy should be included to treatment protocols of stage IV BC. The effects of endocrine, cytotoxic, targeted, or combination treatments in PMBC have been studied in many prospective clinical trials. Despite initial response, mostly such patients develop progressive disease within 12-24 months, the median survival of endocrine nonresponsive or resistant metastatic $B C$ is $18-24$ months, and less than $5 \%$ of patients live 5 years. If a response is observed at all sites, continuation of the effective systemic therapy is reasonable [11].

The role of locoregional therapy in patients with metastatic BC is largely unexplored. There were none prospective randomized trials dealing with this topic. In patients whose tumor is well controlled at distant sites but is progressing locally, local surgery and/or radiotherapy should be discussed. Traditionally, patients with PMBC have limited local treatments and palliative management of uncontrolled local and/or regional disease (in a form of so-called "toilette mastectomy" or lowdose radiotherapy) [2]. There are still open questions regarding the type of breast surgery and indication for radiotherapy. The biological role for removing the primary tumor in a case of disease dissemination is debatable. Several potential advantages have been proposed. By removing the primary tumor, one of the sources of further metastatic spread is eradicated; this risk of reseeding is more relevant with the current improvements in systemic treatments. Data from animal studies suggest that removal of tumor bulk may restore immunocompetence because the primary tumor seems to modulate the immune system through release of immunosuppressive factors. A reduction in the number of cancer cells may also lead to increased efficacy of systemic therapy by decreasing the risk of emergence of chemoresistant cells and by removal of necrotic tumor tissue poorly accessible to drugs. Debulking surgery has been proven clinically effective in other common solid tumors, such as ovarian, colorectal, gastric, renal cancers, and malignant melanoma [11].

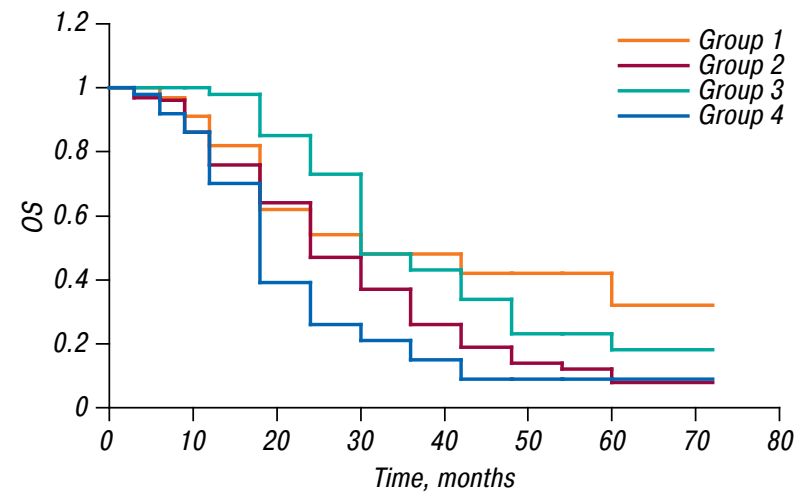

Figure. OS in patients with PMBC analyzed using the Kaplan Meier method

In PMBC, radiotherapy of the primary tumor is frequently combined with breast surgery, making it difficult to discriminate between effects of surgery or radiotherapy alone. In terms of locoregional con- 
trol, primary radiotherapy seems to be as effective as surgery. In a retrospective study by Bourgier et al. [12], radiation alone was compared with surgery of the primary tumor plus radiotherapy. The 3-year OS rates were 39 and 57\%, respectively. Le Scodan et al. [13] presented a retrospective review of patients with $\mathrm{PMBC}$ at the Renee Cancer Center in France. LRT was applied to 320 patients: 249 (78\%) were treated with radiotherapy alone, 41 (13\%) underwent surgery plus radiation, and $30(9 \%)$ were treated only with surgery. The 3-year OS rates were $43.2 \%$ in the group with LRT and $26.7 \%$ in the group without LRT. In our study, 2- and 5 -year OS in patients who gotten radiotherapy were $47 \%$ and $8 \%$, respectively, and 73 and $18 \%$ in patients who received combination of breast surgery and radiation.

Our study showed the LRT of the intact primary tumor in PMBC patients improved median survival time and OS. The pivotal question, which selected patients would benefit from breast surgery concerning survival, cannot be answered by the existing retrospective data by the current meta-analysis. The optimal point of time and the decision for or against breast surgery and radiation depending on the response to systemic therapy remain unclear. Nevertheless, results of multivariable analyses controlling these confounding factors, consistently suggest a survival benefit for optimal LRT of the primary tumor. Some questions still remain unsolved, including which patients could benefit most from LRT and what is its optimal timing and the best systemic cytotoxic treatment regime for these selected patients. Prospective randomization studies are needed to validate these findings. While waiting for data from these studies, LRT for breast primary tumor can be considered as a relatively inexpensive and low morbidity treatment, which can offer a rapid local control and has a potential for impoving patient's survival.

\section{CONCLUSIONS}

The optimal management of stage IVBC is unknown, and thus there is no consensus about the value of surgery in the management of this population. A clinician and a patient may consider surgical resection of the primary tumor in this setting for multiple reasons. LRT of the primary tumor in metastatic BC patients could exert two major effects: the effect of surgery on OS and its effect on local disease control. In patients whose tumor is well controlled at distant sites but progressing locally, local surgery and/or radiotherapy should be discussed.

The results of this study showed the positive impact of LRT on the prognosis of patients with PMBC. However, further research should be aimed on establishing criteria for selecting patients with PMBC for primary tumor LRT (surgery or/and radiotherapy).
This research was presented in part at the Advanced Breast Cancer Third International Consensus Conference, November 5-7, 2015, Lisbon, Portugal.

\section{ACKNOWLEDGEMENTS}

The authors would like to thank the medical personal of Municipal Clinical Oncological Centre for professional work on diagnostic, treatment and taking care of the patients during the research.

\section{CONFLICT OF INTERESTS}

The authors declared no conflict of interests.

\section{REFERENCES}

1. Ferlay J, Soerjomataram I, Ervik M, et al. GLOBOCAN 2012 v1.0, Cancer Incidence and Mortality Worldwide: IARC CancerBase No. 11 [Internet]. Lyon, France: International Agency for Research on Cancer; 2013.

2. Hartmann S, Reimer T, Gerber B, Stachs A. Primary metastatic breast cancer: the impact of locoregional therapy. Breast Care 2014; 9: DOI: 10.1159/000360054.

3. Bulletin of National cancer registry of Ukraine № 16. Cancer in Ukraine, 2013-2014, Kyiv 2015.

4. Bafford AC, Burstein HJ, Barkley CR, et al. Breast surgery in stage IV breast cancer: Impact of staging and patient selection on overall survival. Breast Cancer Res Treat 2009; 115: $7-12$.

5. Harris E, Barry M, Malcolm RK. Meta-analysis to determine if surgical resection of the primary tumour in the setting of stage IV breast cancer impacts on survival. Ann Surg Oncol 2013; 20: 2828-34.

6. Flanigan RC, Salmon SE, Blumenstein BA, et al. Nephrectomy followed by interferon alfa- $2 \mathrm{~b}$ compared with interferon alfa-2b alone for metastatic renal cell cancer. $\mathrm{N}$ Engl J Med 2001; 345: 1655-9.

7. Rosen SA, Buell JF, Yoshida A, et al. Initial presentation with stage IV colorectal cancer: How aggressive should we be? Arch Surg 2000; 135: 530-4.

8. Dauplat J, Le Bouedec G, Pomel C, Scherer C. Cytoreductive surgery for advanced stages of ovarian cancer. Semin Surg Oncol 2000; 19: 42-8.

9. Griffith CT, Parker LM, Lee S, Finkler NJ. The effect of residual mass size on response to chemotherapy after surgical cytoreduction for advanced ovarian cancer: long-term results. Int J Gynecol Cancer 2002; 12: 323-31.

10. Lin SZ, Tong HF, You T, et al. Palliative gastrectomy and chemotherapy for stage IV gastric cancer. J Cancer Res Clin Oncol 2008; 134: 187-92.

11. Pagani O, Senkus E, Wood W, et al. International guidelines for management of metastatic breast cancer: can metastatic breast cancer be cured? J Natl Cancer Inst 2010; 102: 456-63.

12. Bourgier C, Khodari W, Vataire A, et al. Breast radiotherapy as part of loco-regional treatments in stage IV breast cancer patients with oligometastatic disease. Radiother Oncol 2010; 96: 199-203.

13. Le Scodan R, Stevens D, Brain E, et al. Breast cancer with synchronous metastases: survival impact of exclusive locoregional radiotherapy. J Clin Oncol 2009; 27: 1375-81. 